\title{
Mass Spectrometry of Sydnones, Sydnonimines and 5-Imino-oxatriazoles
}

\author{
TAKashi ShIMA, AKIRA OUChIDA AND YutaKa A SAHI
}

(Received 30 March 1969)

Mass spectrometry of mesoionic compounds had not been reported before a recent communication ${ }^{1)}$ on some sydnones. The present authors have independently studied on the fragmentations of some twenty derivatives of sydnones (I-V), sydnonimines (VI-XXI) and 5-imino-oxatriazoles (XXII-XXIV) upon electron impact.

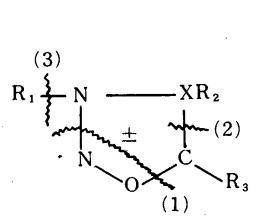

$\mathrm{R}_{3}: \mathrm{O}$

$\mathrm{XR}_{2}: \mathrm{CH}$

$\mathrm{R}_{3}: \mathrm{NH} \cdot$

$\mathrm{HCl}$,

$\mathrm{X}: \mathrm{C}$

$(\mathrm{XVIII}-\mathrm{XX}) \mathrm{R}_{3}: \mathrm{N}$-acyl $\cdot$

$\mathrm{HCl}$,

$\mathrm{XR}_{2}: \mathrm{CH}$

$(\mathrm{XXI})$

$\mathrm{R}_{3}: \mathrm{N}-\mathrm{CO}_{2} \mathrm{C}_{2} \mathrm{H}_{5}, \mathrm{XR}_{2}$ : $\mathrm{CH}$

$(X X I I-X X I V) \cdot \mathrm{R}_{3}: \mathrm{NH} \cdot \mathrm{HCl}, \mathrm{XR}_{2}: \mathrm{N}$

The highest mass peaks of the hydrochlorides are not molecular peaks of the salts $\left(\mathrm{M}^{\prime}\right)$ but of the bases $\left(\mathrm{M}^{\prime}-\mathrm{HCl}\right.$ : M). These bases cleave generally in 3 steps with successive eliminations of $\mathrm{NO} \cdot \mathrm{CR}_{3}$ and $\mathrm{NXR}_{2}$. Base peaks of these compounds examined were mostly due to the substitutents $\left(R_{1}, R_{2}\right.$ or $\left.R_{3}\right)$. Minor fragment peaks containing the mesoionic rings were sometimes observed; $\mathrm{M}-\mathrm{C}_{5} \mathrm{H}_{10}$ in $\mathrm{I}, \mathrm{M}-\mathrm{C}_{3} \mathrm{H}_{6}$ and $\mathrm{M}-\mathrm{C}_{3} \mathrm{H}_{7}$ in VIII, $\mathrm{M}-\left(\mathrm{CH}_{3}\right)_{2} \mathrm{CH}$ and $\mathrm{M}-\mathrm{C}_{6} \mathrm{H}_{10}$ in XVI,
$\mathrm{M}-\mathrm{C}_{3} \mathrm{H}_{6}$ in XVII, $\mathrm{M}-\mathrm{CH}_{3}$ and $\mathrm{M}-\left(\mathrm{CH}_{3}+\right.$ $\mathrm{C}_{6} \mathrm{H}_{10}$ ) in XIX, and $\mathrm{M}-\mathrm{OC}_{2} \mathrm{H}_{5}$ in XXI.

The fragment ions arising through elimination of $\mathrm{NO}$ - from 3-phenyl-or 3 amino-sydnones were more abundant than those from 3-alkylsydnones, probably owing to resonance stabilization of the $\mathrm{M}$-NO- species as following;

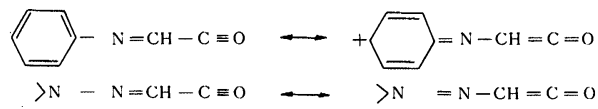

The fragment peaks due to $\mathrm{M}-\mathrm{HNO}$ were observed in the spectra of the imines (VI-XVII) whereas not in the sydnones (I-V) and rarely in the $\mathrm{N}$-acyl-sydnonimines (XVIII-XXI). N-Deuterio derivative of (XVI) gave a $\mathrm{M}-\mathrm{DNO}$ peak at $m / e 178$ instead of the M-HNO peak. Therefore origin of the hydrogen transferred must be the 5 -imino group. The M-30 peak of (XI) can be assigned not to $\mathrm{M}-\mathrm{CH}_{2} \mathrm{O}$ but to $\mathrm{M}-\mathrm{NO}$ - since expected peaks at $m / e 131$ and $m / e ~ 104$ due to further successive eliminations of NO. and $\mathrm{HCN}$ are not observed.

Apart from the peak at $m / e 113$ due to $\mathrm{M}-\dot{\mathrm{N} O} \cdot-\mathrm{HCN}$, (XIV) exhibited an additional peak at $m / e 114$ owing to M-NO- CN fragment, which was observed in the spectrum of $\mathrm{N}$-morpholino-N-nitrosoaminoacetonitrile (XXV). Therefore 5 -imino hydrogen in (XIV) seems to mi-

* Chemical Research Laboratories, Research and Development Division, Takeda Chemical Industries, Ltd., Juso, Osaka, Japan. 
Table 1. Mass Spectra ${ }^{+}$of Sydnones (I-V), Sydnonimines (VI-XXI) and 5-Imino-oxatriazoles (XXII-XXIV).

\begin{tabular}{|c|c|c|c|c|c|c|c|c|c|c|c|c|c|c|c|c|c|}
\hline \multirow[b]{2}{*}{ I } & \multirow{2}{*}{$\frac{\mathrm{R}_{1}}{\mathrm{C}_{6} \mathrm{H}_{13}}$} & \multirow{2}{*}{$\begin{array}{l}\begin{array}{c}\text { Structures } \\
\mathrm{XR}_{2}\end{array} \\
\mathrm{CH}\end{array}$} & \multirow[t]{2}{*}{$\mathrm{R}_{3}$} & \multirow{2}{*}{$\begin{array}{c}\mathrm{M} \\
170 \\
(5)\end{array}$} & \multirow{2}{*}{$\frac{-\mathrm{NO}}{140}$} & \multirow{2}{*}{$\frac{-\mathrm{CR}_{3}}{112 *}$} & \multirow{2}{*}{$\frac{\mathrm{R}_{1}}{85 *}$} & \multicolumn{4}{|c|}{$\begin{array}{l}\text { m/e (Relative intensities) } \\
\quad \text { From } \mathrm{R}_{1}, \underline{\mathrm{R}_{2}}, \underline{\mathrm{R}_{3}}\end{array}$} & \multirow[b]{2}{*}{$\begin{array}{l}100^{a)} \\
(2)\end{array}$} & \multicolumn{3}{|c|}{ Miscellaneous } & & \\
\hline & & & & & & & & $\begin{array}{c}57 \\
(12)\end{array}$ & $\begin{array}{c}55 \\
(8)\end{array}$ & $\begin{array}{c}43 \\
(100)\end{array}$ & $\begin{array}{l}41 \\
(26)\end{array}$ & & $\begin{array}{l}97^{\mathrm{b})} \\
(1)\end{array}$ & & & & \\
\hline II & $\mathrm{C}_{6} \mathrm{H}_{5}$ & $"$ & $" \cdot$ & $\begin{array}{l}162 \\
(16)\end{array}$ & $\begin{array}{l}132 \\
(10)\end{array}$ & $\frac{104}{(88)} *$ & ${ }_{(100)}^{77 *}$ & $\begin{array}{c}51 \\
(40)\end{array}$ & & & & & & & & & \\
\hline III & Cyclohexyl & $"$ & " & $\begin{array}{l}168 \\
(14)\end{array}$ & $\begin{array}{l}138 \\
(1)\end{array}$ & $\begin{array}{l}110 \\
(4)\end{array}$ & $\frac{83}{(75)} *$ & $\begin{array}{c}55 \\
(100)\end{array}$ & $\begin{array}{c}67 \\
(8)\end{array}$ & $\begin{array}{c}41 \\
(70)\end{array}$ & $\begin{array}{l}39 \\
(24)\end{array}$ & & & & & & \\
\hline IV & $\left(\mathrm{CH}_{3}\right)_{2} \mathrm{~N}$ & $"$ & $"$ & $\begin{array}{l}129 \\
(4)\end{array}$ & $\underset{(55)}{99} *$ & $\begin{array}{c}71 \\
(60)\end{array}$ & ${ }_{(90)}^{44} *$ & $\begin{array}{c}42 \\
(94)\end{array}$ & $\begin{array}{c}43 \\
(50)\end{array}$ & $\begin{array}{c}28 \\
(100)\end{array}$ & & & & & & & \\
\hline V & Morpholino. & $"$ & $"$ & $\begin{array}{c}171 \\
(2)\end{array}$ & $\begin{array}{l}141 \\
(26)\end{array}$ & $\begin{array}{c}113 \\
(5)\end{array}$ & $\begin{array}{c}86 \\
(20)\end{array}$ & $\begin{array}{c}56 \\
(100)\end{array}$ & $\begin{array}{c}42 \\
(15)\end{array}$ & & & & & & & & \\
\hline VI & $\mathrm{CH}_{3}$ & $"$ & $\mathrm{NH} \cdot \mathrm{HCl}$ & $\begin{array}{c}99 \\
(53)\end{array}$ & $\begin{array}{c}69 \\
(5)\end{array}$ & $\begin{array}{c}42 \\
(100)\end{array}$ & & & & & & $\left(28^{\mathrm{f})}\right.$ & & & & & \\
\hline VII & $\mathrm{C}_{2} \mathrm{H}_{5}^{\prime}$ & $"$ & $"$ & $\begin{array}{l}113 \\
(64)\end{array}$ & $\begin{array}{l}83 \\
(18)\end{array}$ & $\begin{array}{l}56 \\
(64)\end{array}$ & $\begin{array}{c}29 \\
(40)\end{array}$ & $\begin{array}{c}28 \\
(100)\end{array}$ & & & & $\begin{array}{l}82^{f)} \\
(3)\end{array}$ & & & & & \\
\hline VIII $^{\circ}$ & $\mathrm{C}_{4} \mathrm{H}_{9}$. & $"$ & ': & $\begin{array}{l}141 \\
(35)\end{array}$ & (11) & $\begin{array}{l}84 \\
(26)\end{array}$ & $\begin{array}{l}57 \\
(95)\end{array}$ & $\begin{array}{c}42 \\
(60)\end{array}$ & $\begin{array}{c}41 \\
(100)\end{array}$ & & & $\begin{array}{l}\left.99^{c}\right) \\
(18)\end{array}$ & $\begin{array}{l}98^{\mathrm{d})} \\
(23)\end{array}$ & $\begin{array}{l}\left.69^{e}\right) \\
(49)\end{array}$ & & & \\
\hline IX & $\mathrm{C}_{6} \mathrm{H}_{5}$ & $"$ & $"$ & $\begin{array}{l}161 \\
(8)\end{array}$ & $\frac{131}{(80)} *$ & $\underset{(50)}{104} *$ & $\begin{array}{c}77 \\
(100)\end{array}$ & $\begin{array}{c}51 \\
(24)\end{array}$ & & & & $\begin{array}{l}130^{f)} \\
(8)\end{array}$ & & & & & \\
\hline X & $\mathrm{C}_{6} \mathrm{H}_{5}\left(\mathrm{CH}_{2}\right)_{2}$ & $"$ & $"$ & $\begin{array}{l}189 \\
(2)\end{array}$ & $\begin{array}{l}159 \\
(3)\end{array}$ & $\begin{array}{l}132 \\
(5)\end{array}$ & $\begin{array}{l}105 \\
(13)\end{array}$ & $\begin{array}{l}104 \\
(30)\end{array}$ & $\underset{(100)}{91} *$ & $\begin{array}{l}65 \\
(14)\end{array}$ & $\begin{array}{ll}77 & 51 \\
(7) & (6)\end{array}$ & $\begin{array}{l}158^{\mathrm{f})} \\
(2)\end{array}$ & & & & & \\
\hline XI & $\mathrm{CH}_{3} \mathrm{O} \cdot \mathrm{C}_{6} \mathrm{H}_{4}$ & & $"$ & $\begin{array}{l}191 \\
(3)\end{array}$ & ${(161)^{*}}^{(100}$ & * ${ }_{(40)}^{134} *$ & $=\frac{107}{(23)} *$ & $\begin{array}{c}77 \\
(33)\end{array}$ * & $\begin{array}{c}51 \\
(12)\end{array}$ & $\begin{array}{c}30 \\
(66)\end{array}$ & & $\begin{array}{l}160^{f)} \\
(10)\end{array}$ & & & & & \\
\hline XII & Cyclohexyl & $"$ & $"$ & $\begin{array}{l}167 \\
(13)\end{array}$ & $\begin{array}{l}137 \\
\text { (1) }\end{array}$ & $\begin{array}{l}110 \\
(6)\end{array}$ & $\begin{array}{c}83 \\
(99)\end{array}$ & $\begin{array}{c}67 \\
(28)\end{array}$ & $\begin{array}{c}55 \\
(100)\end{array}$ & $\begin{array}{c}41 \\
(40)\end{array}$ & & & & & & & \\
\hline XIII & $\left(\mathrm{CH}_{3}\right)_{2} \mathrm{~N}$ & " & $"$ & $\begin{array}{l}128 \\
(38)\end{array}$ & $\begin{array}{c}98 \\
(58)\end{array}$ & $\begin{array}{c}71 \\
(50)\end{array}$ & $\begin{array}{c}44 \\
(90)\end{array}$ & $\begin{array}{c}43 \\
(100)\end{array}$ & $\begin{array}{c}42 \\
(90)\end{array}$ & $\begin{array}{l}28 \\
(90)\end{array}$ & & $\begin{array}{c}97^{f)} \\
(40)\end{array}$ & $\begin{array}{l}59^{\mathrm{g})} \\
(40)\end{array}$ & & & & \\
\hline XIV & Morpholino & " & $"$ & $\begin{array}{l}170 \\
(4)\end{array}$ & $\begin{array}{l}140 \\
(24)\end{array}$ & $\begin{array}{l}113 \\
(2)\end{array}$ & $\begin{array}{c}86 \\
(20)\end{array}$ & $\begin{array}{c}56 \\
(65)\end{array}$ & $\begin{array}{c}42 \\
(86)\end{array}$ & $\begin{array}{c}28 \\
(100)\end{array}$ & & $\begin{array}{l}139^{f)} \\
(22)\end{array}$ & $\begin{array}{l}114^{\mathrm{h})} \\
(2)\end{array}$ & $\begin{array}{l}101^{\mathrm{g})} \\
(76)\end{array}$ & & & \\
\hline XV & Morpholino & $\mathrm{CCH}_{3}$ & $\mathrm{NH} \cdot \mathrm{HCl}$ & $\begin{array}{l}184 \\
(19)\end{array}$ & $\begin{array}{l}154 \\
(10)\end{array}$ & $\begin{array}{l}127 * \\
(6)\end{array}$ & * $\begin{array}{c}86 \\
(39)\end{array}$ & $\begin{array}{c}56 \\
(100)\end{array}$ & $\begin{array}{l}42 \\
(24)\end{array}$ & & & $\begin{array}{c}101^{\mathrm{g})} \\
(5)\end{array}$ & $\begin{array}{c}153^{f)} \\
(3)\end{array}$ & & & & \\
\hline XVI & Cyclohexyl & $\mathrm{CCH}\left(\mathrm{CH}_{3}\right)_{2}$ & $"$ & $\begin{array}{l}209 \\
(17)\end{array}$ & $\begin{array}{l}\left.178^{f}\right) \\
(1)\end{array}$ & $\left(\begin{array}{l}152 \\
(4)\end{array}\right.$ & $\begin{array}{c}83 \\
(80)\end{array}$ & $\begin{array}{c}55 \\
(100)\end{array}$ & $\begin{array}{l}41 \\
(53)\end{array}$ & $\frac{43}{(40)}$ & & $\begin{array}{l}166^{\mathrm{i})} \\
(1)\end{array}$ & $\begin{array}{l}163^{\mathrm{j})} \\
(1)\end{array}$ & $\begin{array}{c}135^{\mathrm{k})} \\
(3)\end{array}$ & $\begin{array}{l}1271) \\
(8)\end{array}$ & $\begin{array}{l}\left.97^{m}\right) \\
(8)\end{array}$ & $\begin{array}{c}\left.70^{n}\right) \\
(68)\end{array}$ \\
\hline XVII- & $\left(\mathrm{CH}_{3}\right)_{2} \mathrm{CH}$ & $\mathrm{CC}_{6} \mathrm{H}_{5}$ & " & $\begin{array}{l}203 \\
(16)\end{array}$ & $\begin{array}{c}172^{f)} \\
(3)\end{array}$ & $\begin{array}{l}146 \\
(8)\end{array}$ & $\begin{array}{c}43 \\
(40)\end{array}$ & $\frac{77}{(22)}$ & $\frac{51}{(10)}$ & & & $\begin{array}{l}161^{c)} \\
(11)\end{array}$ & $\begin{array}{l}157^{0)} \\
(4)\end{array}$ & $\begin{array}{l}\left.117^{p}\right) \\
(31)\end{array}$ & $\begin{array}{l}\left.104^{9}\right) \\
(100)\end{array}$ & & \\
\hline XVIII & Morpholino & $\mathrm{CH}$ & $\mathrm{NCHO} \cdot \mathrm{HCl}$ & $\begin{array}{l}198 \\
(14)\end{array}$ & $\begin{array}{l}168 \\
(12)\end{array}$ & $\begin{array}{l}113 \\
(8)\end{array}$ & $\begin{array}{c}86 \\
(18)\end{array}$ & $\begin{array}{c}56 \\
(100)\end{array}$ & $\begin{array}{c}42 \\
(20)\end{array}$ & $\frac{29}{(40)}$ & $\frac{28}{(50)}$ & $\begin{array}{l}\left.140^{r}\right) \\
(40)\end{array}$ & & & & & \\
\hline XIX & Cyclohexyl & $"$ & $\mathrm{NCOCH}_{3} \cdot \mathrm{HCl}$ & $\begin{array}{l}209 \\
(35)\end{array}$ & $\begin{array}{l}179 \\
(-)\end{array}$ & $\begin{array}{l}110 \\
(-)\end{array}$ & $\begin{array}{l}83 \\
(75)\end{array}$ & $\begin{array}{c}55 \\
(86)\end{array}$ & $\begin{array}{c}67 \\
(18)\end{array}$ & $\begin{array}{c}41 \\
(36)\end{array}$ & $\frac{43}{(100)}$ & $\begin{array}{l}194^{\mathrm{s})} \\
(4)\end{array}$ & $\begin{array}{l}\left.137^{t}\right) \\
(62)\end{array}$ & $\begin{array}{l}112^{4)} \\
(11)\end{array}$ & & & \\
\hline $\mathrm{XX}$ & Morpholino & $"$ & $\mathrm{NCOC}_{6} \mathrm{H}_{5} \cdot \mathrm{HCl}$ & $\begin{array}{l}274 \\
\text { (1) }\end{array}$ & $\begin{array}{c}244 \\
(4)\end{array}$ & $\begin{array}{l}113 \\
(-)\end{array}$ & $\begin{array}{l}86 \\
(2)\end{array}$ & $\begin{array}{c}56 \\
(5)\end{array}$ & $\frac{105}{(100)} *$ & $\frac{77}{(30)}$ & $\frac{51}{(8)}$ & $\begin{array}{l}139^{f)} \\
(1)\end{array}$ & & & & & \\
\hline XXI & $"$ & $"$ & $\mathrm{NCO}_{2} \mathrm{C}_{2} \mathrm{H}_{5}$ & $\begin{array}{l}242 \\
(9)\end{array} *$ & $\begin{array}{c}212 \\
(2)\end{array}$ & $\begin{array}{l}113 \\
(7)\end{array}$ & $\begin{array}{l}86 * \\
(42)\end{array}$ & $\begin{array}{c}56 \\
(100)\end{array}$ & $\begin{array}{c}42 \\
(20)\end{array}$ & & & $\begin{array}{l}197^{v)} \\
(4)\end{array}$ & $\begin{array}{l}168^{w)} \\
(6)\end{array}$ & $\begin{array}{l}\left.140^{x}\right) \\
(44)\end{array}$ & $\begin{array}{l}\left.101^{g}\right) \\
(4)\end{array}$ & & \\
\hline XXII & $\mathrm{C}_{6} \mathrm{H}_{3}$ & $\mathrm{~N}$ & $\mathrm{NH} \cdot \mathrm{HCl}$ & $l^{162}{ }^{*}$ & $\begin{array}{c}132 \\
(6)\end{array}$ & $\begin{array}{l}105 \\
(20)\end{array}$ & ${ }_{(100)}^{77}{ }^{*}$ & $\begin{array}{c}51 \\
(35)\end{array}$ & & & & $\begin{array}{l}131 \mathrm{f}) \\
(2)\end{array}$ & & & & & \\
\hline XXIII & o. $\mathrm{CH}_{3} \cdot \mathrm{C}_{6} \mathrm{H}_{4}$ & & " & $\begin{array}{l}176 \\
(2)\end{array}$ & $\begin{array}{l}146 \\
(6)\end{array}$ & $\frac{119}{(12)} *$ & ${ }^{*} \underset{(100)}{91} *$ & $\begin{array}{c}65 \\
(26)\end{array}$ & & & & $\begin{array}{l}145^{f)} \\
(2)\end{array}$ & & & & & \\
\hline XXIV & $\mathrm{p} \cdot \mathrm{Cl} \cdot \mathrm{C}_{6} \mathrm{H}_{4}$ & $"$ & $"$ & $\begin{array}{l}196 \\
(2)\end{array}$ & $\begin{array}{l}166 \\
(10)\end{array}$ & $\begin{array}{l}139 \\
(28)\end{array}$ & ${ }_{(100)}^{111} *$ & $\begin{array}{c}75 \\
(40)\end{array}$ & & & & $\begin{array}{l}165^{f)} \\
(6)\end{array}$ & & & & & \\
\hline$X X V$ & & No) $\mathrm{CH}_{2} \mathrm{CN}$ & & $\begin{array}{l}170 \\
(-)\end{array}$ & $\begin{array}{l}140 \\
(13)\end{array}$ & $\begin{array}{l}114^{\mathrm{h})} \\
(8)\end{array}$ & $\begin{array}{l}86 \text { * } \\
(16)\end{array}$ & $\begin{array}{c}56 \\
(64)\end{array}$ & $\begin{array}{c}42 \\
(90)\end{array}$ & $\begin{array}{c}28 \\
(100)\end{array}$ & & $\begin{array}{l}139^{f)} \\
(26)\end{array}$ & & & & & \\
\hline
\end{tabular}

†) With a Hitachi RMU-6D double-focusing mass spectrometer, direct inlet system, ionizing energy of $70 \mathrm{eV}$, ion source temperature below $150^{\circ} \mathrm{C}$, evaporation temperature below decomposition temperature of each compound.

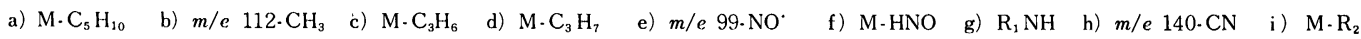

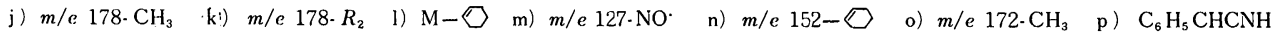

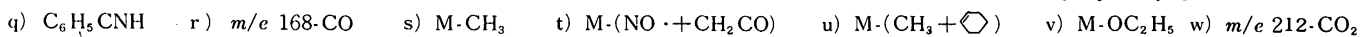
x) $m /$ e $168 \cdot \mathrm{C}_{2} \mathrm{H}_{4}$

*: Metastable peak. 
grate partially to the carbon at 4 position during the measurement.

A fragment peak at $m / e 101$ arising from (XIV) and (XV) is assigned to iminomorpholium ion. The 5- $\mathrm{N}$-deuterio derivative of $(\mathrm{XV})$ gave a peak at $\mathrm{m} / \mathrm{e}$ 102 instead of the peak at $m / e 101$. 3-Dimethylaminosydnonimine.(XIII) showed a fragment peak of $\left(\mathrm{CH}_{3}\right)_{2} \mathrm{~N}^{+}=$ $\mathrm{NH}$ at $m / e 59$, whereas sydnones and sydnonimines with 5 -N-acyl or 3-alkyl did not undergo such, a fragmentation. Therefore these fragments will be formed by migration of the 5 - $\mathrm{N}$-hydrogens to the nitrogen at 3 position. The much abundance of these species will be caused by high stability of the quarternary ammonium structures.

The ethyl group in (XXI) seems to migrate to $5 \mathrm{~N}$ with eliminating carbon dioxide after expulsion of NO: The hydrogens of $5 \mathrm{~N}$-formyl in (XVIII) and $5 \mathrm{~N}$ acetyl in (XIX) transfer also to the $5 \mathrm{~N}$ with eliminations of $\mathrm{CO}$ and $\mathrm{CH}_{2} \mathrm{CO}$, respectively. A $5 \mathrm{~N}$-benzoyl derivative (XX) did not show'such a migration.

Fragmentations of 5-imino-oxatriazoles (XXII-XXIV) resemble to those of sydnonimines.

\section{Acknowledgement}

The authors are grateful to Takeda Chemical Industries, Ltd. for the permission of the publication. Thanks are also due to Dr. K. Masuda for his offering the materials.

\section{Reference}

1) J. H. Bowie, R. A. Eade and J. C. Earl, Aust. J. Chem. 21, 1665 (1968). 